\title{
Streit und Lärm in der Stadt
}

\section{Klosterreform und Stadt}

Streit und Lärm im Umfeld der Klosterreform des 15. Jahrhunderts - das ist etwas überraschend. ${ }^{1}$ Denn die Melker Reform war eher eine ruhige, schriftgestützte Rückbesinnung aus dem Geist der Benediktsregel. ${ }^{2}$ Eher eine Abkehr vom Lärm der Welt als ein Kampfruf gegen deren Zustände. Allerdings lagen die Augsburger Klöster in einer Stadt, vor deren Leben sich die Mönche in den Klostermauern nicht einfach abschließen konnten. Und in vielen Fällen wollten sie das auch gar nicht. Denn die Mönche waren mit der Stadt auf vielfältige Weise verbunden: Durch die Familie, durch die eigene Geschichte, durch Freunde und Unterstützer, durch die Nachbarschaft. Tatsächlich bietet ja die Geschichte monastischer Reformen im Mittelalter, die immer Rückbesinnungen sein wollten, in der historischen Realität eine gut hörbare Tonspur von lauten Kontroversen, intoniert etwa von Hirsauer Mönchen, die im 11. Jahrhundert durch Schwaben zogen und für die Reform der Kirche stritten, oder von Bernhard von Clairvaux, der das Erscheinungsbild des großen Cluny im 12. Jahrhundert in scharfen Worten geißelte, oder von umherziehenden Bettelmönchen, deren Predigten auf den Plätzen der Stadt die Bewohner in großen Zahlen anlockten und dabei für erheblichen Lärm sorgten. ${ }^{3}$ Die Bußpredigten des Johannes Capistrano 1454 im Augsburger Fronhof, die Tausende anlockten, wider die sündigen Vergnügungen, sorgten für gewaltige Aufläufe. Von Streit berichten die Anwesenden weniger, zu dem Capistrano aber sicher aufgerufen hätte, wenn die Juden nicht schon ein paar Jahre zuvor aus der Stadt vertrieben worden wären. Er hatte ein einschlägiges Register. ${ }^{4}$

Das Leben der Mönche in der Stadt, auch das geistliche und liturgische Leben, war eng mit den Bürgern der Stadt verbunden. Und das 15. Jahrhundert hatte als eine Phase des besonderen Selbstbewusstseins der Augsburger Bürgerschaft ein erhebliches Streitpotential in einem bürgerlich-geistlichen Spannungsfeld. ${ }^{5}$

1 Der Beitrag behält seinen Vortragscharakter bei, die Nachweise sind auf das Wesentliche beschränkt.

2 Vgl. zuletzt: Glaßner 2013,75-92; Klugseder 2012, 169-209.

3 Für einen groben Überblick vgl. etwa Lawrence 2003.

$4 \mathrm{Zu}$ Johannes Capistrano in Augsburg vgl. die Augsburger Annalen des Johannes Frank, in: Die Chroniken der Schwäbischen Städte 5 1896, 283-340, 303-305.

5 Vgl. dazu den Beitrag von Rolf Kießling in diesem Band. 
Als markante Punkte seien etwa der lange Streit um die Besetzung des Bistums zu Beginn des Jahrhunderts genannt, der Konflikt Peter von Schaumbergs mit der Stadt um die bischöfliche Rolle in der Stadtherrschaft nach seiner Erhebung zum Kardinal 1450, oder der notorische Konflikt um die Aufnahme von Bürgersöhnen in das Domkapitel. ${ }^{6}$ Johannes Janota und Werner Williams haben vor 20 Jahren die Forschungen über das literarische Leben in Augsburg in einem Band zusammengestellt, der das spät erwachte, aber erkennbar laikale Selbstbewusstsein zeigt. $^{7}$ Geistlich-bürgerliche Spannungen waren an der Tagesordnung, wie Hektor Mülich um die Mitte des 15. Jahrhunderts vermerkte: „Item unser thumherren und unser pfaffen waren zu allen zeiten unseres schadens fro und stritten täglich wider das gemain volck mit worten."8

Nun betraf die Melker Reform die lebenslustigen Domherren und die Augsburger Pfarrer als Weltgeistliche nicht direkt, aber in Zeiten, in denen um die Rolle der Geistlichkeit in der Stadt gestritten wurde, berührten diese Konflikte auch die Mönche in St. Ulrich und Afra und die Augustinerchorherren in St. Georg oder Hl. Kreuz. ${ }^{9}$ Die Spannungen umfassten ein gewisses Spektrum. Der Auftakt der kurzen annalistischen Notizen des Johannes Frank, der 1451 Novize im Kloster St. Ulrich und Afra wurde und der im folgenden Jahr, in dem in Rom die letzte mittelalterliche Kaiserkrönung vorgenommen wurde, die Profess ablegte, ist an mönchsspezifischer Dramatik kaum zu übertreffen. „Item da man zalt nach Christus gepurd 1430 jar an Sant Antonius abent (i.e. Mitte Januar), da ran ain junge frau auf dem Lech herab an die nidern pruck und was angeschmitt mit henden und füssen und hals und sas und het in der hand ains münichs haupt“. ${ }^{10}$ Ein dramatisches Bild - eine junge Frau mit Ketten an Händen, Füssen und Hals sitzt an der Brücke nach Lechhausen und wiegt den (wahrscheinlich blutigen) Kopf eines Mönches in ihrem Schoß. Aber wir müssen doch nüchterner hinzufügen, dass es dabei bleiben muss. Wir wissen nichts über das Gesicht dieses Mönchs. Und wir wissen auch nichts über den Ausdruck auf dem Gesicht der jungen Frau. Wir wissen tatsächlich nichts über die Hintergründe des Geschehens. Etwas genauer wissen wir dagegen Bescheid über die Umstände einer Lärmentwicklung im Zentrum der Stadt etwa 10 Jahre später, die direkt auf das Reformgeschehen in Augsburg

6 Für einen einführenden Überblick vgl. Gottlieb et al. 1985, Krüger 2005, 31-43; Krüger 2012, 62-70.

7 Janota/Williams-Krapp 1995.

8 Chronik des Hektor Mülich 1348-1487, in: Die Chroniken der schwäbischen Städte 3 1892, 104.

9 Für eine Übersicht über die geistlichen Häuser in Augsburg in dieser Phase vgl. Liebhart 1985, 193-201.

10 Johannes Franks Augsburger Annalen, in: Die Chroniken der schwäbischen Städte 5 1896, 283-340, 295. 
zurückging. Diesmal kommt eine Gruppe von Frauen ins Bild. Wir sehen sie nur durch Fenster in der Mauer. Es geht um das Dominikanerinnenkloster St. Katharina. Die Fenster in der Mauer waren durch Gitter verschlossen, aber durch die Gitterstäbe konnten die Nonnen mit den Leuten auf der Straße sprechen. Nun waren Maurer aufgezogen, brachen die Gitter heraus und schickten sich an, die Öffnungen zuzumauern. Doch sie hatten nicht mit der Entschlossenheit der Frauen im Kloster gerechnet. Burkhard Zink, der die Szene ausführlich wiedergibt, schreibt, dass die Baumaßnahme die Frauen so zornig machte, dass sie mit Stangen und Bratspießen durch die Öffnung stachen und die Handwerker an ihrer Arbeit hinderten. Sie wichen der Gewalt. „Kainer torst da ichts machen.“ Da standen die städtischen Handwerker und wagten nicht, ihre Arbeit fortzusetzen. Es war die Klosterreform, die die Nonnen von Sankt Katharina zum wütenden Widerstand anspornte. Und auch die Tonspur wurde belegt. Denn als die Stadt ihre Knechte schickte, um die Handwerker zu schützen, da läuteten die Nonnen die Glocken, um die Übergriffe des Augsburger Rates und seiner Handlanger anzuprangern. Auch dies ein eindrucksvolles Bild. „An dröhnen die Klöppel und ließen nicht Zeit dem erregten Metall“11 auf der Strecke zwischen Rathaus und Sankt Ulrich, die inzwischen dichter bewohnt war, so dass die Menschen zusammenströmten, um dem lärmenden Streit um den Abschluss der Nonnen von Sankt Katharina von der städtischen Population beizuwohnen. Das Geschehen hatte in diesem Fall einen Hintergrund, den uns Burkard Zink nicht verschweigt. Es ging darum, dass man die „closterfrauen von Sant Katharina gereformieret und verschloßen hat“, denn vor dieser Maßnahme um 1440 war die Besuchsregelung im Kloster vergleichsweise liberal gewesen: „es mochten zu in gan ir guet freund und wer wolt, sie mochten auch bei ainer weil wol aufgan $\mathrm{zu}$ irn freunden." ${ }^{22}$ Diese Praxis hatte nach Burkard Zink im Jahre 1441 bereits 41 Jahre angedauert. Jetzt hielt ein neuer Geist Einzug. Man kann sich vorstellen, dass manche Tochter aus gutem Hause diese Entwicklung mit Missbilligung sah, da sie sich mit einem gewissen Recht darauf berufen konnte, unter anderen Bedingungen in das Kloster eingetreten zu sein. Man fand indes einen Kompromiss, mit dem alle Beteiligten leben konnten. Die Nonnen riefen den Bischof zu Hilfe, der eine diplomatische Lösung aushandelte. Die Nonnen sollten (zunächst für zwei Jahre) künftig die Regel - den „orden“ - einhalten und auf die Außenkontakte verzichten. Wer dies nicht tun mochte, sollte das Kloster verlassen können und sein ein-

11 Mann 1974, 9.

12 Das dramatische Reformgeschehen in Sankt Katharina wird geschildert von Burkard Zink in seiner Chronik, in: Die Chroniken der schwäbischen Städte 2 1866, 103-104. 
gebrachtes Gut zurückerhalten. Diese Frauen wurden einem anderen Kloster zugewiesen. ${ }^{13}$ Dieser Einzug der Reform in das Augsburger Klosterleben ging dem Einzug der Melker Reform in der Stadt um einige Jahre voraus und die Kraft hinter dieser letztlich disziplinarischen Maßnahme war offenbar der Rat der Stadt, also ein Laiengremium. Der Befund bestätigt die anfänglich festgestellte Verbindung von Kloster und umgebender Welt. Das Geschehen könnte uns daran erinnern, dass bei einer früheren geistlichen Reform im 11. Jahrhundert die Augsburger Domkleriker bitter über die Forderung der Laien nach der Ehelosigkeit der Priester geklagt hatten ${ }^{14}$ und wir könnten so den Erzählstrang von einer geistlichen Welt in der Krise fortsetzen, die sich nur durch äußeren Zwang von ihrer Bigotterie abwendete. Tatsächlich aber gibt es dafür wenige Gründe, und es wird Zeit, dass wir nach einem ausführlicheren anekdotischen Auftakt einem gewissen analytischen Ernst Raum geben. Denn der nicht sonderlich fromme Burkard Zink räumte ein, dass nach der bischöflichen Kompromissformel die Nonnen mit der neuen Disziplin wohl zufrieden waren und nur wenige das Kloster verließen. ${ }^{15}$

Tatsächlich war die Lautstärke, die Klöster und Kirchen in Augsburg in der Stadt entfalten konnten, in Konfliktsituationen nicht das Hauptproblem. Die Stadt hatte mit der Autorität über die Sturmglocke die Möglichkeit, selber hörbar Aufmerksamkeit einzufordern - wobei sich die Angehörigen der Bürgergemeinde freilich darüber einigen mussten, wer den Zugang zu dieser Glocke hatte, wer die Bürger zu den Waffen rufen durfte. Die Glocken der Klöster und der Augsburger Kirchen riefen im 15. Jahrhundert - anders als bei der Stadtzerstörung 1132 - nicht mehr zu den Waffen. Ihre Signale konnten das Zeichen zum Konflikt sein - die Quellen drücken das in der eigentümlichen Formulierung aus, die Glocken läuteten „ueber“ jemanden, d.h. sie machten eine Missetat publik. ${ }^{16}$ Nur konnte man diese Anklage dem Klang der Glocke im Detail nicht entnehmen. Die Glocke konnte nur zur Bekanntmachung einer Anklage oder eines Urteils rufen. Das taten Geistliche und auch die Stadt gleichermaßen. Wirklich konfrontativ aber war es, wenn die Glocken schwiegen und die Geistlichen „nicht sangen“. Das war die Formulierung für die schwerste geistliche Sanktion gegen die Stadt - das Interdikt, die Aussetzung des Gottesdienstes und der Sakramentenspendung. ${ }^{17}$ Im ge-

13 Burkard Zink in seiner Chronik, in: Die Chroniken der schwäbischen Städte 2 1866, 104.

14 Annales Augustani, in: Monumenta Germaniae Historica Scriptores 3 1839, 123-136, 129.

15 Burkard Zink in seiner Chronik, in: Die Chroniken der schwäbischen Städte 2 1866, 104.

16 Burkard Zink in seiner Chronik, in: Die Chroniken der schwäbischen Städte 2 1866, 104; vgl. dazu allgemein: Haverkamp 2002, 277-313.

17 Vgl. Kaufhold 1994, 62-67. 
wissen Sinne das disziplinarische Gegenstück zum Ablass, der im späten Mittelalter so populär war. In dem Streit um den Augsburger Bischofssitz, der 1413 nach der Wahl des Augsburger Domkustos zum neuen Bischof anhob, weil die Stadt diesen Anselm von Nenningen entschieden ablehnte, kam es in der Folge zur Verhängung des Interdikts über Augsburg, oder wie man damals sagte: „die pfaffen hörten auf zu singen."18 Sie tauften keine Kinder mehr und beerdigten die Toten nicht mehr nach kirchlichem Ritus. Vor den Rat zitiert, der sie darauf verwies, dass Papst und König mit Friedrich von Grafeneck einen anderen Bischof ernannt hätten, weigerten sie sich, den Gottesdienst wieder aufzunehmen. Das große abendländische Schisma dauerte noch immer an, es gab mehr als einen Papst, und die Loyalitäten waren nicht einfach zu verteilen. Also mussten die Geistlichen die Stadt verlassen. Um die Versorgung mit den Sakramenten und die Aufrechterhaltung der Gottesdienste zu ermöglichen, wandte sich der Rat an die Klöster der Stadt. Die Mönche erbaten drei Tage Bedenkzeit, „und sie bedachten sich und sangen und lasen und tetten, als sie dann vor getan hetten. "19 $\mathrm{Zu}$ ruhig sollte es in der Stadt nicht sein.

\section{Keine einfachen Lagerbildungen}

Für das Verhältnis geistlicher Institutionen und politischer Kräfte in der Stadt im 15. Jahrhundert eignen sich einfache Lagerbildungen nicht. $\mathrm{Zu}$ unterschiedlich waren die Interessen von Bischof und adligem Domkapitel, von Bettelorden, klassischen Benediktinern und Augustinerchorherren in einer Stadt, die dramatisch wuchs und deren Sozialstruktur mit einer kleinen reichen Oberschicht und der großen Zahl armer Handwerker und Wollarbeiter für erhebliche politische Spannungen sorgte, in denen sich die Augsburger Klöster und Konvente positionieren mussten. ${ }^{20}$ Wilfried Ehbrecht hat in verschiedenen Studien auf die Bedeutung städtischer Klöster als Orte und Räume der Verhandlung und des Ausgleichs von städtischen Konfliktparteien verwiesen. ${ }^{21}$ In Augsburg nahmen diese Aufgabe wohl vor allem die Barfüßer wahr. Deren Refektorium wurde in den schweren Unruhen über die Besteuerung mit Ungeld in den letzten Jahren des 14. Jahrhunderts zum Anlaufpunkt für die Vertreter der armen Zünfte, wie Weber, Bäcker

18 Burkard Zink. Chronik, in: Die Chroniken der schwäbischen Städte 2 1866, 76-77.

19 Burkard Zink. Chronik, in: Die Chroniken der schwäbischen Städte 2 1866, 77.

20 Vgl. dazu Gottlieb et al. 1985, 187-193; 208-213; vgl. außerdem Kießling 1971.

21 Vgl. zum Beispiel Ehbrecht 2001. 
und Schuster. ${ }^{22}$ Nach ihren wütenden Protesten kamen die Vertreter des Rates in den Barfüßerkonvent, um den künftigen Verzicht auf neues Ungeld zu beurkunden. Das Ungeld war eines der großen städtischen Konfliktthemen dieser Phase, vielleicht das größte, und die Vereinbarung hielt nicht sehr lange. Dennoch ist dieses Geschehen bei den Barfüßern wichtig, denn es bezeichnet einen Wandel, der sich schon in der Zunftrevolte zeigte und der die Konfliktführung des 15. Jahrhundert entscheidend so prägte, dass wir neben dem schon gehörten Lärm so viel anderen gar nicht zu hören bekommen. Und das, obwohl der Chronist angesichts der Ungeldunruhen 1397 schreibt: „daz volk wolt immer sin streit haben.“23 Und dieses Volk erschien zur Bekräftigung seiner Forderung nach der Abschaffung der Weinbesteuerung bewaffnet zu den Verhandlungen. Trotz dieser Konfliktbereitschaft eines erheblichen armen Bevölkerungsteils und trotz der Tatsache, dass die reichen Augsburger Bürger im Grunde adligen Idealen nachstrebten, wie sich etwa an den Fuggern hundert Jahre später zeigte, und dass die jungen Domherren aus adligen Familien kamen - mit einer Lebensordnung, in der die Waffen eine besondere Bedeutung hatten -, blieb die Zahl gewaltsamer Übergriffe auf Geistliche oder von Geistlichen überschaubar. ${ }^{24}$ Es sind gelegentliche Bluttaten, wie der Mord an dem Pfarrer von St. Stephan, Hans Berner, an Weihnachten 1426, oder der Überfall auf das Haus des Domdekans Leonhard Gessel an Palmsonntag 1462, der sich mit dem Söldner Endis Truchseß überworfen hatte, worauf dieser einige Gefährten mobilisierte und in das Haus des Dekans eindrang, die Schlösser und Türen aufbrach und alles verwüstete. Der Lärm blieb indes nicht verborgen, und die städtischen Autoritäten rückten an, stoppten das Treiben und verhafteten die Wüstlinge. ${ }^{25}$ Der Domdekan war ein reicher Mann, und sein Besitz rief sicher einigen Neid hervor. Wenn man sich nun erinnert, wie noch im späten 13. Jahrhundert die Bürger die Häuser der Domherren im Konflikt überfielen und niederbrannten, ohne dass die Täter zur Rechenschaft gezogen worden wären, so ist der Wandel deutlich erkennbar. ${ }^{26}$ Die Stadt duldete solche Übergriffe nicht mehr. Und die Täter wären noch schwerer bestraft worden, wären nicht Männer

22 Vgl. dazu etwa Kaufhold 2013, 10-18.

23 Chronik von 1368 bis 1406, in: Die Chroniken der schwäbischen Städte 11865, 110.

24 Zu den Fuggern vgl. Häberlein 2006. Zum Augsburger Domkapitel zuletzt Krüger 2006, $27-$ 48.

25 Burkard Zink. Chronik, in: Die Chroniken der schwäbischen Städte 2 1866, 268-270; Hektor Mülich. Chronik 1348-1487, in: Die Chroniken der schwäbischen Städte 31892, 181-182; vgl. auch Johannes Franks Augsburger Annalen, in: Die Chroniken der schwäbischen Städte 5 1896, 330331.

26 Vgl. Krüger 2012, 64-65 mit Abb. 5. 
des Markgrafen von Brandenburg an dem Überfall beteiligt gewesen, der sich mit großem Gefolge in Augsburg befand.

Spannungen zwischen Geistlichen und Bürgern gab es auf vielen Feldern, wenn man an die Erneuerung einer Reihe von Ansprüchen aus der alten bischöflichen Stadtherrschaft denkt, die Peter von Schaumberg in der Mitte des 15. Jahrhunderts vortrug, und die die Bürger entschieden zurückwiesen - in diese Phase fällt ja auch der schon zitierte Befund von Hektor Mülich über die Feindseligkeit der Domherren - aber tatsächliche Handgreiflichkeiten kamen im Grunde nur noch auf einem besonderen Feld vor: dem der Baulichkeiten an der Stadtmauer. Hierüber hatte es schon mit dem bereits genannten Anselm von Nenningen im ausgehenden 14. Jahrhundert Streit gegeben. Die Stadt duldete keine geistlichen Mauerbauten oder Bauten an der Mauer in ihrer Zuständigkeit. Dabei ging es wiederholt um Anbauten, oder 1447 sogar um einen Turm an der Mauer, den der Rat für einsturzgefährdet hielt und abreißen wollte, was die Geistlichen, auf deren Grund der Turm stand, nicht gestatteten. Also verschafften sich die städtischen Bauleute mit Gewalt Zugang zum Turm und rissen ihn nieder. Menschen kamen dabei nicht zu Schaden. Da diese Bauten an der Stadtmauer, ähnlich wie auch der Stadtpyr, an verschiedenen Orten in der Stadt eine durchaus symbolische Bedeutung hatten, drückte sich in dieser ,Gewalt gegen Sachen' schon ein klarer Deutungsanspruch aus. ${ }^{27}$ Der Rat beanspruchte die städtische Hoheit. Die Geistlichkeit protestierte, erreichte aber nicht viel. Gelegentlich flackerte bei diesen Konflikten Gewalt auf, aber der eigentliche Streit wurde auf einer anderen Bühne ausgetragen, auf der Ebene schriftlicher Dokumente. Sie waren lange das Privileg adliger Geistlicher und herrschaftsnaher Juristen gewesen. Auch das Stadtrecht, das mit tätiger Hilfe der Franziskaner zu einem Instrument des patrizischen Rates wurde, war zunächst nur für einen exklusiven Kreis wichtiger Familien erreichbar. Das zeigte sich in der Zunftrevolte, als die einfachen Zünfte die Aushändigung des Stadtbuches verlangten, das ihnen bis dahin kaum zugänglich war. ${ }^{28}$ Diese Forderung wurde durch einen friedlichen, aber bewaffneten, Aufmarsch unterstützt. 1397 wiederholte sich dieses Szenario in den Ungeldunruhen. Die Zünfte der Weber, Bäcker, Schuster und die anderen waren zwar bewaffnet, aber sie verlangten als Sicherheit dafür, dass es künftig keine Verbrauchssteuern

27 Vgl. zu diesen Streitigkeiten und Übergriffen um die Mitte des 15. Jahrhunderts: Kaufhold 2009, 52-71.

28 Chronik von 1368 bis 1400, in: Die Chroniken der schwäbischen Städte 1 1865, 21-125, 21: „sie wölten der stat puch ein nehmen und prieff und der stat insigel und die schlüzzel zu der sturmgloggen.“ 
mehr auf den Wein geben sollte, einen Brief, also eine Urkunde. ${ }^{29}$ Das bedeutet, dass um 1400 in Augsburg, dessen Sozialstruktur Ähnlichkeiten mit heutigen Drittweltländern hatte, die soziale Ordnung in den Augen aller Beteiligten, nicht nur in den Augen einer privilegierten Oberschicht, durch geschriebenes Recht gesichert werden sollte. ${ }^{30}$ Als es 1466 wegen des Ungelds wieder zu starken Unruhen kam, da bildete man schließlich einen Ausschuss aus Vertretern aller 17 Zünfte und der Herren. Dieser sollte einen Kassensturz machen und die Finanzen der Stadt einer gründlichen Prüfung unterziehen - „dieselben 18 man sollen niedersitzen und vollen gewalt haben zu verhören und ze lesen alle freihait, alle brief, statpuech und alles, das diese stat hat [...]“31

Hier sind wir nun in der Zeit, in der die Melker Reform im Kloster St. Ulrich und Afra verstärkt Einzug hält. In dieser Phase der Stadtgeschichte wird auch in den mitunter scharfen sozialen Konflikten der Stadt die schriftliche Überlieferung zu einem Maßstab des Handelns. Diese schriftliche Überlieferung wurde nun sorgfältig geprüft. Das heißt nicht, dass es immer gerechter zuging. Aber der Austrag der Konflikte fand eine überwiegend juristisch-schriftliche Form. Mit dem Blick auf das Reformgeschehen sollten wir diesen Wandel feststellen. Das Umfeld der Reform hatte sich erkennbar verändert. Frühere benediktinische Reformer in Cluny, Hirsau und Citeaux hatten sich durch die strikte Orientierung an Texten als Trägern der Reform von ihrer weitgehend analphabetischen Umgebung abgehoben. Das galt für den Adel und für die Bauern. Denn lesen und schreiben konnten auch die Adligen in der Regel nicht, und die Klostermauern trennten ihre agrarische Welt von der Welt der Mönche. Die Melker Reform musste sich in Augsburg nun auch in einer Umwelt bewähren, deren städtische Lebensordnung außerhalb des Klosters ebenfalls auf geschriebenen Texten aufbaute. Ein Teil der alten Distinktion ging dadurch verloren. Und die Klostermauern wurden durchlässiger, denn die zunehmend schriftlich formulierten Positionen in den städtischen Konflikten konnten durch eben diese Schriftform verstärkten Einzug in das Klosterleben halten. Die Reformer standen damit vor einer komplexer werdenden Herausforderung.

29 Urkundenbuch der Stadt Augsburg II. Hrsg. Meyer Nr. 800: 277-278, Nr. 804: 282-283.

30 Zur Sozialstruktur Augsburgs in dieser Zeit vgl. Gottlieb et al. 1985, 187-193.

31 Zink 1866, 118-121, hier 119. 


\section{Literatur}

„Annales Augustani”, in: Monumenta Germaniae Historica Scriptores. Bd. 3, hrsg. von Georg Wilhelm Pertz. Hannover 1839. 123-136.

„Chronik von 1368 bis 1406“, in: Die Chroniken der schwäbischen Städte. Augsburg 1. Leipzig 1865.

Ehbrecht, Wilfried: Konsens und Konflikt. Skizzen und Überlegungen zur älteren Verfassungsgeschichte deutscher Städte, hrsg. von Peter Johanek. Köln 2001.

Frank, Johannes: „Annalen“, in: Die Chroniken der Schwäbischen Städte. Augsburg 5. Leipzig 1896. 283-340, 303-305.

Glaßner, Christine: „Stift Melk und die Melker Reform“, in: Die benediktinische Klosterreform im 15. Jahrhundert, hrsg. von Franz-Xaver Bischof und Martin Turner. Berlin 2013. 75-92.

Gottlieb, Gunther/Baer, Wolfram/Becker, Joseph (Hrsg.): Geschichte der Stadt Augsburg von der Römerzeit bis zur Gegenwart. Stuttgart 1985.

Häberlein, Mark: Die Fugger. Geschichte einer Augsburger Familie (1367-1650). Stuttgart 2006.

Haverkamp, Alfred: „,....an die große Glocke hängen‘. Über Öffentlichkeit im Mittelalter“, in: Gemeinden, Gemeinschaften und Kommunikationsformen im hohen und späten Mittelalter. Festgabe zur Vollendung des 65. Lebensjahres, hrsg. von Michael Matheus, Friedhelm Burgard und Clemens Lukas. Trier 2002. 277-313.

Janota, Johannes/Williams-Krapp, Werner (Hrsg.): Literarisches Leben in Augsburg im 15. Jahrhundert. Tübingen 1995.

Kaufhold, Martin: Gladius spiritualis. Das päpstliche Interdikt in der Regierungszeit Ludwigs des Bayern (1324-1347). Heidelberg 1994.

Kaufhold, Martin: „Prügeleien am Stadtpyr. Ein zerrissener Mantel und die politischen Kämpfe der Reichsstadt (um 1450)“, in: Augsburg im Mittelalter, hrsg. von Martin Kaufhold. Augsburg 2009. 52-71

Kaufhold, Martin: „Die Franziskaner im mittelalterlichen Augsburg“, in: Barfuß vor St. Max. Von der mittelalterlichen Klosterkirche der Franziskaner zur Pfarrkirche St. Maximilian, hrsg. von Melanie Thierbach. Augsburg 2013. 10-18.

Kießling, Rolf: Bürgerliche Gesellschaft und Kirche in Augsburg im Spätmittelalter. Ein Beitrag zur Strukturanalyse der oberdeutschen Reichsstadt. Augsburg 1971.

Klugseder, Robert: „Die Auswirkungen der Melker Reform auf die liturgische Praxis der Klöster", in: Studien und Mitteilungen zur Geschichte des Benediktinerordens und seiner Zweige 123 (2012): 169-209.

Krüger, Thomas: „Peter von Schaumberg“, in: Lebensbilder aus dem Bistum Augsburg, hrsg. von Manfred Weitlauff. Augsburg 2005. 31-43.

Krüger, Thomas: „Die Hausherren des Doms und sein funktionsgeschichtlicher Wandel“, in: Der Augsburger Dom im Mittelalter, hrsg. von Martin Kaufhold. Augsburg 2006. 27-48.

Krüger, Thomas: „Gewalt und Recht. Bürgerlich - Klerikale Streitkultur im mittelalterlichen Augsburg“, in: Städtische Kultur im Mittelalterlichen Augsburg, hrsg. von Martin Kaufhold. Augsburg 2012. 62-70.

Lawrence, Clifford Hugh: Medieval monasticism. Forms of religious life in Western Europe in the middle ages. 3. Aufl. London 2003. 
Liebhart, Wilhelm: „Stifte, Klöster und Konvente in Augsburg“, in: Geschichte der Stadt Augsburg von der Römerzeit bis zur Gegenwart, hrsg. von Gunther Gottlieb, Wolfram Baer und Joseph Becker. Stuttgart 1985. 193-201.

Mann, Thomas: Der Erwählte. 2., durchgesehene Aufl. Frankfurt a. M. 1974.

Mülich, Hektor: „Chronik 1348-1487“, in: Die Chroniken der schwäbischen Städte 3. Leipzig 1892.

Urkundenbuch der Stadt Augsburg II, hrsg. von Christian Meyer. Augsburg 1878.

Zink, Burkard: „Chronik“, in: Die Chroniken der schwäbischen Städte 2. Leipzig 1866. 